\title{
计算机网络信息安全在大数据下的防护措施探究
}

\author{
季 烨 \\ 天津市教育委员会教育信息化管理中心，天津 300191
}

[摘要]科学技术的发展为各个行业的发展壮大创造了良好的基础, 计算机网络技术的发展促进了大数据技术的进步, 并且这 项技术已经被人们引用到了多个领域之中, 取得了十分良好的成绩。计算机网络信息安全与大数据的运用效果存在密切的关 联, 但是因为计算机网络信息安全极易受到外界各种因素的影响而诱发危险, 不但会造成信息的泄露, 并且会导致严重的经 济损失。要想切实的对上述问题加以解决, 最为重要的就是需要从各个角度出发提升人们的计算机网络信息安全防护意识, 并综合实际情况来制定预防方案, 从根本上对网络信息的安全性和准确性加以保证。

[关键词]大数据时代; 计算机网络信息安全; 防护措施

DOI：10.33142/sca.v3i2.1848 中图分类号: TP393.08 文献标识码: A

\section{Research on the Protection Measures of Computer Network Information Security under Big Data}

JI Ye

Tianjin Municipal Education Commission Education Information Management Center, Tianjin, 300191, China

\begin{abstract}
The development of science and technology has created a good foundation for the development of various industries, the development of computer network technology has promoted the progress of big data technology, and this technology has been used in many fields, and achieved very good results. The information security of computer network is closely related to the application effect of big data. However, because the information security of computer network is easily affected by various external factors, it will not only cause information leakage, but also lead to serious economic losses. In order to solve the above problems, the most important thing is to enhance people's awareness of computer network information security protection from all angles, and to make prevention plans based on the actual situation, so as to fundamentally guarantee the security and accuracy of network information.
\end{abstract}

Keywords: big data era; computer network information security; protective measures

引言

社会的稳步发展, 推动了大数据技术的稳步发展, 从而为我国综合国力的提升带来了良好的机遇。计算机是当下大 数据时代发展必不可少的重要部分, 其可以借助网络信息化系统针对网络信息加以统一收集、存储、分析和输出, 从而 为大数据时代网络信息化的发展提供良好的基础。就现如今计算机网络信息安全性实际情况来说, 整体水平并没有达到 完善的状态, 所以还需要我们针对其中的问题进行有效的解决, 从而为我国计算机网络化的发展创造良好的基础。

\section{1 大数据的基本概况}

经过调查分析我们发现, 大数据往往借助以往传统的数据传输软件进行信息的获取、处理和输送都是非常困难的。 就大数据的实质来看, 其就是拥有巨大容量的数据库, 将简单形式的数据信息借助多元化的形式来加以整合, 最为突 出的特征就是种类多, 数量巨大, 价值低、成本花费少等等 ${ }^{[1]}$ 。在科学技术整体水平快速提升的影响下, 使得大数据被 人们大范围的运用到了生活和工作的各个角落之中, 在这个过程中人们对于大数据的内涵也有了进一步的认识。大数 据在各个领域中的运用就是借助大数据高速传递、运用以及存储从而创建一个覆盖范围较为广泛的网络平台。在大数 据快速发展的形势下, 人们的工作模式以及生活理念也发生了明显的变化。总的来说, 大数据时代的到来为人类社会 的发展带来了良好的机遇。近年来大数据的发展越发的成熟, 并且在社会经济发展中起到积极的推动作用, 世界中各 大互联网商家都是借助大数据来对整个企业的发展进行综合规划, 在推动企业的稳步健康发展方面起到了积极的影响 ${ }^{[2]}$ 。

\section{2 计算机网络信息安全的重要性}

因为计算机网络中的信息具有一定的共享性并且各个终端设备的分布并不集中, 从而导致极易遭到诸多危险病毒 或者是黑客的攻击。针对那些会对网络安全造成危害的因素, 我们务必要对网络的安全性加以综合分析考虑。以信息 
类企业为例来说, 在企业对信息数据的实时性和准确性越发关注的形势下, 我们可以创设专门的网站以及信息平台对 企业运营涉及到的各项信息进行统一的收集, 如果不能切实的创设安全防护系统, 那么就会为危险因素的入侵提供机 会, 最终就会发生信息泄露的情况, 这样对于企业的稳步发展是非常不利的。所以企业要安排专业人员来加强计算机 网络信息安全管理，针对其中存在隐性风险加以预判，并制定有效的预防和解决方案。

\section{3 大数据时代背景下影响计算机网络安全的重要因素}

\section{1 大数据时代背景下网络开放性所引发的安全问题}

在当下大数据的时代中, 计算机网络在实际运行过程中起到了良好的开放性的影响作用, 正是因为这一巨大的影 响作用也为计算机网络的发展造成了诸多的限制, 极易引发网络危险 ${ }^{[3]}$ 。在整个计算机网络运行过程中, 尤其是用户借 助计算机来实施信息的传递和共享的时候, 往往不会设立专门的限制条件, 这种开放式的运行模式极易造成信息泄露 的情况, 为一些不法分子提供机会进行信息的窃取从而实施犯罪行为。这样就会对使用者的安全造成一定的损害, 并 且会对社会的和谐发展产生诸多的阻碍。

\section{2 使用者操作不当所引发的安全问题}

在实际运用计算机网络来进行信息传递或者是共享的时候, 使用者往往都是依据自身的主观意识来进行实践操作 的, 这样往往会在计算机网络中埋下诸多的危险隐患, 再加上使用者自身的安全意识较差, 或者是不具备良好的安全 防范意识, 也不具备良好的计算机操作技能, 从而导致计算机的操作方式方法存在诸多的疏漏, 这样也会为不法分子 提供机会进行信息的窃取。所以, 使用者在进行口令或者是密码设置的时候, 务必要遵照规范标准来开展工作, 这样 才能有效的规避后续网络安全问题的发生 ${ }^{[4]}$ 。

\section{3 计算机病毒攻击所引发的安全问题}

计算机网络遭受病毒的攻击可以说是最为常见的问题, 这样不但会对计算机的正常运行造成一定的阻碍, 甚至会 发生信息泄露的情况。在当下大数据时代中, 因为计算机网络具有一定的公开性的特征, 所以也为病毒的攻击创造而 来良好的基础, 从而会导致病毒在较短的时间内能够完成大范围的侵入。就计算机病毒来说, 自身具备良好的隐蔽性, 并且还具备一定的存储性能, 所以在将计算机网络加以实践运用的时候, 如果遇到病毒的入侵使用者是很难及时的发 现的。最后病毒会侵入到计算机网络程序之中, 从而造成大幅度的病毒传播, 病毒快速、大范围的传播所造成的不良 后果是非常严重的, 往往也会对计算机网络的使用者的信息安全造成威胁 ${ }^{[5]}$ 。

\section{4 黑客入侵所引发的安全问题}

计算机遇到黑客的入侵是引发网络安全问题的主要根源, 通常黑客的入侵就其性质来说只是人为性质的一种安全 问题。黑客借助一些专业的方式和技术进入到用户的计算机程序之中, 从而实现对计算机内部信息窃取的目的, 这样 就会造成被侵入的计算机内信息数据丢失、泄露的情况。这种人为侵入计算机的操作往往会造成计算机网络系统的整 体㿈疾, 最终会对人们的生活和工作带来诸多的不良影响。

\section{4 大数据时代计算机网络信息安全防护措施}

在当下大数据的时代中, 计算机设备是信息传递的基础, 网络信息的安全性往往会受到诸多外界不良因素的影响, 所以无法得以保障, 这就需要我们从预防环节入手增强计算机网络信息安全防护整体性能的水平, 排除计算机网络安 全中存在的危险隐患。

\section{1 网络信息安全管理防护工作的加强}

计算机网络信息安全的防护工作的落实需要在实际运用计算机的时候全面的做好网络信息安全管理防护工作, 借助 专业计算机技术为计算机的安全运转提供必要的保障, 并且要针对计算机网络信息系统进行定期专业检查, 加大力度实 施网络安全管理工作，从根本上保证计算机网络信息的安全性，最大限度的避免危险因素对网络正常运行造成损害。

\section{2 重视计算机安全系统的应用}

计算机安全系统是确保计算机程序能够高效、稳定运行的重要基础, 在当下大数据时代中, 网络信息安全是人们 最为关心的问题, 可以借助在计算机中安设安全系统的方式来提升计算机的安全性能。现如今防火墙是人们使用最为 
频繁的一种计算机安全防护方式, 为了保证计算机网络信息的安全性, 可以创建计算机安全系统, 针对侵入的病毒进 行有效的抵挡。其次, 网络管理工作人员要针对计算机安全系统进行定期的维护和升级, 从根本上促进计算机网络信 息系统整体的安全性能。

\section{3 强化计算机网络管控有效性}

现如今, 电子计算机的适用范围在不断的扩展, 在这种形势下, 计算机网络信息安全问题越发的眼中, 为了彻底 的扭转这一局面, 我们需要从网络管理的角度入手, 加大力度对计算机网络进行全面的管控, 尽可能的规避网络风险 的发生。计算机网络管理人员也要不断的提升自身的专业能力和安全防护意识, 完善计算机网络环境, 促进计算机网 络信息的整体安全性。

\section{4 加强信息安全防范意识}

计算机网络信息化能够为社会的稳步发展创造良好的基础, 但是企业要想保证自身的稳定发展, 需要对计算机网络 信息化进行全面的管控。当下计算机网络大范围的运用, 不法人员利用计算机网络来窃取商业机密的情况越来越严重, 所以企业需要创设适合自己的网络防护系统, 避免各项信息的外泄, 并且还需要利用有效的专业技术来增强计算机系统 数据的防护效果和力度，结合实名认证提高对计算机系统的保护作用，更好的达到计算机网络信息安全防护目的。

\section{结论}

综上所述, 大数据时代, 计算机网络技术迅速发展, 尽管有效的推动了网络信息的共享效率, 但是也带来了信息 泄露的风险, 最终会引发诸多的经济损失。所以我们需要对计算机网络的安全性加以重点关注, 制定专门的管理制度, 从宏观上保护计算机网络发展, 为社会稳定发展创造良好的基础。

\section{[参考文献]}

[1]刘梦飞.大数据背景下计算机网络信息安全风险及防护措施 [J]. 现代工业经济和信息化, 2017, 7 (21) : 59-61.

[2]汤应.大数据背景下的计算机网络信息安全及防护措施 [J]. 现代工业经济和信息化, 2018, 8(01): 50-51.

[3]李伟洪.大数据时代计算机网络信息安全与防护措施 $[\mathrm{J}]$.电子技术与软件工程, 2019, 9 (08): 201 .

[4] 欧高权.大数据时代下计算机网络信息安全问题 [J].电子技术与软件工程, 2019, 8(10): 200 .

[5]王世轶.大数据时代计算机网络信息安全与防护措施 [J].计算机产品与流通, 2019,8(07): 58.

作者简介: 季烨 (1983.9-), 男, 天津市人, 大学学历, 主要从事教育信息化项目规划、建设和管理工作（涉及数据 中心基建, 服务器, 存储, 虚拟化, 云计算, 云安全, 数据灾难备份管理及研究)。 